\title{
The statistical dynamics of turbulent Rossby wave flow over topography
}

\author{
T. J. O’Kane* J. S. Frederiksen ${ }^{\dagger}$ \\ (Received 13 December 2004, revised 5 July 2005)
}

\begin{abstract}
The statistical dynamics of Rossby wave turbulence is examined by comparing direct numerical simulation of the vorticity form of the 2-D Navier-Stokes equation with a non-Markovian statistical closure theory for inhomogeneous flow over mean topography. The quasi-diagonal direct interaction approximation closure theory is formulated for the interaction of mean fields, Rossby waves and inhomogeneous turbulence over topography on a generalized $\beta$-plane. The competing effects of nonlinear waves at the large scales and fully developed turbulence at the small scales is examined by comparing closure theory with ensemble averaged results from direct numerical simulation at resolution $k=48$ for circularly truncated wavenumber space. This work builds
\end{abstract}

${ }^{*}$ CSIRO Atmospheric Research, Aspendale, Australia. mailto: Terence.0'Kane@csiro.au

${ }^{\dagger}$ CSIRO Atmospheric Research, Aspendale, Australia. mailto: Jorgen. Frederiksen@csiro.au

See http://anziamj.austms.org.au/V46/CTAC2004/Okan for this article, (C) Austral. Mathematical Soc. 2005. Published July 27, 2005. ISSN 1446-8735 
on the low resolution $\beta$-plane studies of Frederiksen and O'Kane (2005) and extends the high resolution $f$-plane studies of O'Kane and Frederiksen (2004) to incorporate waves. We also examine the performance of a computationally efficient restart or cumulant update procedure at moderate Reynolds number in the presence of waves.

\section{Contents}

1 Introduction

2 2-D flow on a generalized $\beta$-plane

3 QDIA closure equations

4 Results and discussion

5 Conclusion

C716

References

C718

\section{Introduction}

The development of modern closures based on renormalized perturbation theory has its origin in the pioneering work of Kraichnan [3] who developed the Eulerian direct interaction approximation (DIA) for homogeneous turbulence. This closure and related Markovianized versions such as the eddy-damped quasi-normal Markovian model (EDQNM) have been successfully applied to a variety of important problems including subgrid-scale parameterizations for eddy viscosity and stochastic backscatter and the study of the statistics of the predictability of homogeneous turbulent flows (see [2] and references therein). 
Recently Frederiksen [1] formulated a quasi-diagonal DIA closure to extend Kraichnan's homogeneous DIA closure to general inhomogeneous turbulence interacting with mean flows and topography (QDIA). The work of Frederiksen [1] was important in that it elucidated the role of the eddytopographic and eddy-mean field interactions enabling parameterizations of the stress due to the interaction of subgrid scale eddies with retained scale topography. Such parameterizations are essential for realistic simulations of ocean circulations and are also lacking in atmospheric general circulation models. O'Kane and Frederiksen [7] examined the performance of the QDIA and a more computationally tractable variant termed the cumulant update QDIA (CUQDIA). They found in their experiments that the QDIA for inhomogeneous $f$-plane two-dimensional turbulence over random topography has similar performance to the DIA for homogeneous two-dimensional turbulence, that it is only a few times more computationally intensive than the DIA for homogeneous turbulence and that a one-parameter regularized version of the QDIA termed the regularized QDIA (RQDIA), in which transfers are localized, is in excellent agreement with DNS at all scales.

Frederiksen and O'Kane [2] generalized the QDIA closure theory to the interaction of Rossby wave turbulence with mean fields and topography on a generalized $\beta$-plane which includes a term corresponding to the solid body rotation vorticity on the sphere that is found to be significant for the structure of the dispersion relations of Rossby waves in the presence of mean flows, for the statistical mechanics of Rossby wave turbulence and for closure theory. Frederiksen and O'Kane [2] further presented low resolution C16 (circularly truncated wavenumber space at $k=16$ ) studies of the closure performance for flow over an isolated conical mountain and for observed northern hemisphere flows. In this paper we examine the performance of the closure at much higher resolution $(\mathrm{C} 48)$ where the effects of waves at the large scales acts to suppress the exchange of energy and enstrophy between the large and small scales where turbulent eddy-eddy interactions dominate. These higher resolution results enable comparison to the high resolution $f$-plane studies of O'Kane and Frederiksen [7] for turbulent flow over topography. We are 
particularly interested in the performance of the cumulant update or restart procedure $[5,6]$ at high resolution when waves are present.

In Section 2, we state the barotropic vorticity equation for flow over topography and Rossby wave turbulence on a generalized $\beta$-plane and in the presence of a large scale flow $U$. We also discuss the form-drag equation for the large scale flow $U$. We use a generalized form of the $\beta$-plane equations for the small-scales and the large scale flow $U$ that allows the closure equations to be written in compact form with the sums over wave number extended to include a zero wavenumber component that incorporates the large scale flow term. In Section 3 we summarize the QDIA equations that include the off-diagonal covariance matrix and non-Gaussian terms.

In Section 4 we then consider the generation of Rossby waves when large scale turbulent flow interacts with single realization mean topography with random phase and amplitude appropriate for the study of atmospheric flows. We examine the performance of the QDIA closure in comparison with ensemble averaged direct numerical simulation (DNS) at moderate resolution $(k \leq 48)$ and moderate large scale Reynolds number $(\approx 305)$.

\section{2-D flow on a generalized $\beta$-plane}

The differential rotation rate plays an important role in the interaction of mean flows, turbulence and topography in many geophysical fluid dynamics contexts. Here we include this effect through the $\beta$-plane approximation generalized to include a term representing the solid body rotation vorticity of the corresponding spherical geometry problem. We represent the full stream function $\Psi=\psi-U y$, where $U$ is a large-scale east-west flow and $\psi$ represents the small scales. The evolution equation for two-dimensional motion of the small scales over a mean topography on a generalized $\beta$-plane is described 
by the barotropic vorticity equation

$$
\frac{\partial \zeta}{\partial t}=-J\left(\psi-U y, \zeta+h+\beta y+k_{0}^{2} U y\right)+\hat{\nu} \nabla^{2} \zeta+f^{0} .
$$

Here $f^{0}$ is the forcing, $\hat{\nu}$ the viscosity,

$$
J(\psi, \zeta)=\frac{\partial \psi}{\partial x} \frac{\partial \zeta}{\partial y}-\frac{\partial \psi}{\partial y} \frac{\partial \zeta}{\partial x}
$$

is the Jacobian, and $k_{0}$ is a wavenumber that specifies the strength of the large-scale vorticity corresponding to the solid body rotation on a sphere. The vorticity is the Laplacian of the stream function $\zeta=\nabla^{2} \psi$. We assume that the variation in the topography $(\Delta H)$ is small, and define $h$ to be the scaled spatial variation of the height of the topography relative to the total depth. The form-drag equation for the large-scale flow $U$ is the same as on the standard $\beta$-plane. With the inclusion of relaxation towards the state $\bar{U}$ it takes the form

$$
\frac{\partial U}{\partial t}=\frac{1}{S} \int_{S} h \frac{\partial \psi}{\partial x} d S+\alpha(\bar{U}-U)
$$

Here, $\alpha$ is a drag coefficient and $S$ is the area of the surface $0 \leq x \leq 2 \pi$, $0 \leq y \leq 2 \pi$. In the absence of forcing and dissipation, Eqs. (1) and (2) together conserve kinetic energy and potential enstrophy.

The standard $\beta$-plane vorticity equation is obtained by setting $k_{0}^{2}$ to zero. However, note that there is a one-to-one correspondence between the generalized $\beta$-plane equation and that for flow on the sphere in the presence of a solid body rotation contribution. The non-dimensional barotropic vorticity equation can be derived by choosing a length scale of $a / 2$, half the earth's radius, and a time scale of $\Omega^{-1}$, the inverse of the earth's angular velocity. We derive the corresponding spectral space equations by representing each of small-scale terms by a Fourier series where

$$
\zeta_{\mathbf{k}}(t)=\frac{1}{(2 \pi)^{2}} \int_{0}^{2 \pi} d^{2} \mathbf{x} \zeta(\mathbf{x}, t) \exp (-i \mathbf{k} \cdot \mathbf{x})
$$


and $\mathbf{x}=(x, y), \mathbf{k}=\left(k_{x}, k_{y}\right), k=\left(k_{x}^{2}+k_{y}^{2}\right)^{1 / 2}$ and $\zeta_{-\mathbf{k}}$ is conjugate to $\zeta_{\mathbf{k}}$.

The spectral equations for the small-scales on the generalized $\beta$-plane can be combined into a compact form by defining suitable interaction coefficients, representing the large-scale flow as a component with zero wavenumber and extending the sums over wavenumbers. We represent the barotropic vorticity equation (1) in the spectral form

$$
\begin{gathered}
\left(\frac{\partial}{\partial t}+\nu_{0}(\mathbf{k}) k^{2}\right) \zeta_{\mathbf{k}}(t)=\sum_{\mathbf{p} \in \mathbf{T}} \sum_{\mathbf{q} \in \mathbf{T}} \delta(\mathbf{k}+\mathbf{p}+\mathbf{q})\left[K(\mathbf{k}, \mathbf{p}, \mathbf{q}) \zeta_{-\mathbf{p}} \zeta_{-\mathbf{q}}\right. \\
\left.+A(\mathbf{k}, \mathbf{p}, \mathbf{q}) \zeta_{-\mathbf{p}} h_{-\mathbf{q}}\right]+f_{\mathbf{k}}^{0}
\end{gathered}
$$

Here $f_{\mathbf{k}}^{0}$ is the forcing and the complex $\nu_{0}(\mathbf{k})$ is related to the viscosity $\hat{\nu}$ and the intrinsic Rossby wave frequency $\omega_{\mathbf{k}}$ by

$$
\nu_{0}(\mathbf{k}) k^{2}=\hat{\nu} k^{2}+i \omega_{\mathbf{k}}
$$

where $\omega_{\mathbf{k}}=-\beta k_{x} / k^{2}$. Also we define $\zeta_{-\mathbf{0}}=i k_{0} U, \zeta_{\mathbf{0}}=\zeta_{-\mathbf{0}}^{*}$ and introduce a term $h_{-0}$ that we take to be zero but which could more generally be related to a large-scale topography. We define $f_{\mathbf{0}}^{0}$ and $\nu_{0}\left(\mathbf{k}_{0}\right)$ by $f_{\mathbf{0}}^{0}=\alpha \bar{\zeta}_{\mathbf{0}}$ and $\nu_{0}\left(\mathbf{k}_{0}\right) k_{0}^{2}=\alpha$ respectively. Thus the spectral form of the barotropic vorticity Eq. (4) holds for all $\mathbf{k}$ in the set $\mathbf{T}=\mathbf{R} \cup \mathbf{0}$.

Note that $U$ is real and we define $\zeta_{0}$ to be imaginary. This is done to ensure that all the interaction coefficients that we use are purely real. It is then possible to extend the sums over $\mathbf{p}$ and $\mathbf{q}$ to include the vector $\mathbf{0}$, to define appropriate real interaction coefficients and map the $\beta$-plane problem into the same form as the $f$-plane problem. Note that we distinguish between $\mathbf{0}$ and $\mathbf{- 0}$ in this representation and these components are complex conjugate, as is the case for the small-scale components with oppositely signed wave vectors. The interaction coefficients needed in Eq. (4) are defined by 


$$
\begin{aligned}
A(\mathbf{k}, \mathbf{p}, \mathbf{q}) & =-\gamma\left(p_{x} \hat{q_{y}}-\hat{p_{y}} q_{x}\right) / p^{2}, \\
K(\mathbf{k}, \mathbf{p}, \mathbf{q}) & =\frac{1}{2}[A(\mathbf{k}, \mathbf{p}, \mathbf{q})+A(\mathbf{k}, \mathbf{q}, \mathbf{p})] \\
& =\frac{\gamma}{2}\left[p_{x} \hat{q_{y}}-\hat{p_{y}} q_{x}\right]\left(p^{2}-q^{2}\right) / p^{2} q^{2}, \\
\delta(\mathbf{k}+\mathbf{p}+\mathbf{q}) & = \begin{cases}1, & \text { if } \mathbf{k}+\mathbf{p}+\mathbf{q}=0, \\
0, & \text { otherwise. }\end{cases}
\end{aligned}
$$

Our definitions of the interaction coefficients are generalized to include the zero wave vector as any of the three arguments by specifying

$$
\begin{aligned}
& \gamma= \begin{cases}-k_{0} / 2, & \text { if } \mathbf{k}=\mathbf{0}, \\
k_{0}, & \text { if } \mathbf{q}=\mathbf{0} \text { or } \mathbf{p}=\mathbf{0}, \\
1, & \text { otherwise; }\end{cases} \\
& \hat{q}_{y}= \begin{cases}1, & \text { if } \mathbf{k}=0 \text { or } \mathbf{p}=0 \text { or } \mathbf{q}=0, \\
q_{y}, & \text { otherwise; }\end{cases} \\
& \hat{p}_{y}= \begin{cases}1, & \text { if } \mathbf{k}=0 \text { or } \mathbf{p}=0 \text { or } \mathbf{q}=0, \\
p_{y}, & \text { otherwise. }\end{cases}
\end{aligned}
$$

Note that

$$
K(\mathbf{k}, \mathbf{p}, \mathbf{q})+K(\mathbf{p}, \mathbf{q}, \mathbf{k})+K(\mathbf{q}, \mathbf{k}, \mathbf{p})=0
$$

for all $\mathbf{k}, \mathbf{p}$ and $\mathbf{q}$ including the zero vectors. With these definitions of the interaction coefficients it can also be shown that Eq. (2) for the large scales can be written in the form Eq. (4).

\section{QDIA closure equations}

The method of deriving the QDIA closure equations [1] and its variants the CUQDIA and RCUQDIA [7] with $\mathbf{k}$ in the set $\mathbf{T}$, has been described in detail 
elsewhere [2]. Here we very briefly state the governing QDIA equations. Suppose we have an ensemble of flows satisfying the spectral barotropic vorticity equation and we express the vorticity $\zeta_{\mathbf{k}}$ and forcing $f_{\mathbf{k}}^{0}$ in terms of their ensemble means, denoted by \langle\rangle , and the deviations from the ensemble mean, denoted by ^:

$$
\begin{aligned}
& \zeta_{\mathbf{k}}=\left\langle\zeta_{\mathbf{k}}\right\rangle+\hat{\zeta}_{\mathbf{k}}, \\
& f_{\mathbf{k}}^{0}=\left\langle f_{\mathbf{k}}^{0}\right\rangle+\hat{f}_{\mathbf{k}}^{0} .
\end{aligned}
$$

The equation for the ensemble mean can then be written

$$
\begin{aligned}
\left(\frac{\partial}{\partial t}+\nu_{0}(\mathbf{k}) k^{2}\right)\left\langle\zeta_{\mathbf{k}}\right\rangle=\sum_{\mathbf{p}} & \sum_{\mathbf{q}} \delta(\mathbf{k}+\mathbf{p}+\mathbf{q}) \\
\times[ & K(\mathbf{k}, \mathbf{p}, \mathbf{q})\left\{\left\langle\zeta_{-\mathbf{p}}\right\rangle\left\langle\zeta_{-\mathbf{q}}\right\rangle+C_{-\mathbf{p},-\mathbf{q}}(t, t)\right\} \\
& \left.+A(\mathbf{k}, \mathbf{p}, \mathbf{q})\left\langle\zeta_{-\mathbf{p}}\right\rangle h_{-\mathbf{q}}\right]+\left\langle f_{\mathbf{k}}^{0}\right\rangle
\end{aligned}
$$

and the equation for the two-point cumulant is defined in terms of the deviation from the mean $\hat{\zeta}$ by

$$
C_{-\mathbf{p},-\mathbf{q}}(t, s)=\left\langle\hat{\zeta}_{-\mathbf{p}}(t) \hat{\zeta}_{-\mathbf{q}}(s)\right\rangle .
$$

Throughout this section $\mathbf{p}$ and $\mathbf{q}$ both range over the set $\mathbf{T}$.

Thus, we see from Eq. (14) that to determine the mean field we need an equation for the two-point cumulant $C_{-\mathbf{p},-\mathbf{q}}(t, t)$. However, the cost of computing the full covariance matrix is prohibitive at any reasonable resolution [4]. The quasi-diagonal DIA closure equations instead express the offdiagonal two-point cumulant and response functions in terms of the diagonal elements. The resulting equations for the mean field, two-point cumulant and response functions are expressed entirely in terms of the diagonal elements of the two-point cumulant and response functions and are computationally much more efficient than the general inhomogeneous closure equations [4].

The QDIA approach uses renormalized perturbation theory to derive the following first order expression for the off-diagonal elements of the covariance 
matrix:

$$
\begin{aligned}
C_{\mathbf{k},-1}\left(t, t^{\prime}\right)= & \int_{t_{0}}^{t} d s R_{\mathbf{k}}(t, s) C_{\mathbf{l}}\left(s, t^{\prime}\right) \\
& \times\left[A(\mathbf{k},-\mathbf{l}, \mathbf{l}-\mathbf{k}) h_{(\mathbf{k}-\mathbf{l})}+2 K(\mathbf{k},-\mathbf{l}, \mathbf{l}-\mathbf{k})\left\langle\zeta_{(\mathbf{k}-\mathbf{l})}(s)\right\rangle\right] \\
& +\int_{t_{0}}^{t^{\prime}} d s R_{-\mathbf{l}}\left(t^{\prime}, s\right) C_{\mathbf{k}}(t, s) \\
& \quad \times\left[A(-\mathbf{l}, \mathbf{k}, \mathbf{l}-\mathbf{k}) h_{(\mathbf{k}-\mathbf{l})}+2 K(-\mathbf{l}, \mathbf{k}, \mathbf{l}-\mathbf{k})\left\langle\zeta_{(\mathbf{k}-\mathbf{l})}(s)\right\rangle\right] \\
& +R_{\mathbf{k}}\left(t, t_{0}\right) R_{-\mathbf{l}}\left(t^{\prime}, t_{0}\right) \tilde{K}_{\mathbf{k},-\mathbf{l}}^{(2)}\left(t_{0}, t_{0}\right)
\end{aligned}
$$

where $\tilde{K}_{\mathbf{k},-1}^{(2)}\left(t_{0}, t_{0}\right)$ is the contribution to the off-diagonal covariance matrix at initial time $t_{0}$ [7]. Similarly, the off-diagonal elements of the response function, which measures the change in the vorticity perturbation due to an infinitesimal change in the forcing, that is

$$
R_{\mathbf{k}, \mathbf{l}}\left(t, t^{\prime}\right)=\left\langle\frac{\delta \hat{\zeta}_{\mathbf{k}}(t)}{\delta \hat{f}_{\mathbf{l}}^{0}\left(t^{\prime}\right)}\right\rangle,
$$

may be written in purely diagonal terms as in [1, Eq. (A.12)]. We also use the abbreviations

$$
C_{\mathbf{k}}\left(t, t^{\prime}\right)=C_{\mathbf{k},-\mathbf{k}}\left(t, t^{\prime}\right) ; \quad R_{\mathbf{k}}\left(t, t^{\prime}\right)=R_{\mathbf{k}, \mathbf{k}}\left(t, t^{\prime}\right) .
$$

Then, using Eq. (16) in Eq. (14), we obtain the mean-field equation to second order in renormalized perturbation theory.

The expression for the diagonal two-time cumulant in terms of two- and three-point terms is

$$
\begin{aligned}
& \left(\frac{\partial}{\partial t}+\nu_{0}(\mathbf{k}) k^{2}\right) C_{\mathbf{k}}\left(t, t^{\prime}\right) \\
& =\sum_{\mathbf{p}} \sum_{\mathbf{q}} \delta(\mathbf{k}+\mathbf{p}+\mathbf{q}) A(\mathbf{k}, \mathbf{p}, \mathbf{q}) C_{-\mathbf{p}-\mathbf{k}}\left(t, t^{\prime}\right) h_{-\mathbf{q}}
\end{aligned}
$$




$$
\begin{aligned}
& +\sum_{\mathbf{p}} \sum_{\mathbf{q}} \delta(\mathbf{k}+\mathbf{p}+\mathbf{q}) K(\mathbf{k}, \mathbf{p}, \mathbf{q}) \\
& \times\left[\left\langle\zeta_{-\mathbf{p}}(t)\right\rangle C_{-\mathbf{q}-\mathbf{k}}\left(t, t^{\prime}\right)+C_{-\mathbf{p}-\mathbf{k}}\left(t, t^{\prime}\right)\left\langle\zeta_{-\mathbf{q}}(t)\right\rangle+\left\langle\hat{\zeta}_{-\mathbf{p}}(t) \hat{\zeta}_{-\mathbf{q}}(t) \hat{\zeta}_{-\mathbf{k}}\left(t^{\prime}\right)\right\rangle\right] \\
& +\int_{t_{0}}^{t^{\prime}} d s F_{\mathbf{k}}^{0}(t, s) R_{-\mathbf{k}}\left(t^{\prime}, s\right)
\end{aligned}
$$

where $F_{\mathbf{k}}^{0}(t, s)=\left\langle\hat{f}_{\mathbf{k}}^{0}(t) \hat{f}_{\mathbf{k}}^{0 *}(s)\right\rangle$ is the random forcing. To close this equation we also need an expression for the three-point cumulant and this is derived in the same way as for the DIA closure for homogeneous turbulence:

$$
\begin{aligned}
& \left\langle\hat{\zeta}_{-\mathbf{l}}(t) \hat{\zeta}_{(\mathbf{l}-\mathbf{k})}(t) \hat{\zeta}_{\mathbf{k}}\left(t^{\prime}\right)\right\rangle \\
& =2 \int_{t_{0}}^{t^{\prime}} d s K(\mathbf{k},-\mathbf{l}, \mathbf{l}-\mathbf{k}) C_{-\mathbf{l}}(t, s) C_{(\mathbf{l}-\mathbf{k})}(t, s) R_{\mathbf{k}}\left(t^{\prime}, s\right) \\
& \quad+2 \int_{t_{0}}^{t} d s K(-\mathbf{l}, \mathbf{l}-\mathbf{k}, \mathbf{k}) R_{-\mathbf{l}}(t, s) C_{(\mathbf{l}-\mathbf{k})}(t, s) C_{\mathbf{k}}\left(t^{\prime}, s\right) \\
& \quad+2 \int_{t_{0}}^{t} d s K(\mathbf{l}-\mathbf{k},-\mathbf{l}, \mathbf{k}) R_{(\mathbf{l}-\mathbf{k})}(t, s) C_{-\mathbf{l}}(t, s) C_{\mathbf{k}}\left(t^{\prime}, s\right) \\
& \quad+R_{-\mathbf{l}}\left(t, t_{0}\right) R_{(\mathbf{l}-\mathbf{k})}\left(t, t_{0}\right) R_{\mathbf{k}}\left(t, t_{0}\right) \tilde{K}_{-\mathbf{l},(\mathbf{l}-\mathbf{k}), \mathbf{k}}^{(3)}\left(t_{0}, t_{0}, t_{0}\right),
\end{aligned}
$$

where $\tilde{K}_{-\mathbf{l},(\mathbf{l}-\mathbf{k}), \mathbf{k}}^{(3)}\left(t_{0}, t_{0}, t_{0}\right)$ allows for non-Gaussian initial conditions. Similarly the equation for the diagonal two-time response function $R_{\mathbf{k}}\left(t, t^{\prime}\right)$ [1, Eq. 3.8] contains the three-point (DIA) [3] and two-point (QDIA) [1] terms.

The numerical approach that we have followed is the same as that used in references $[7,2]$. The forward time step is achieved via a predictor-corrector scheme while the time-history integrals in the cumulant and mean-field equations are discretized by the trapezoidal rule. The QDIA is a second order renormalized closure and thus contains all higher order terms to some approximation. The closure and DNS models are formulated for discrete spectra on the doubly periodic domain and run over half the wavenumber space by invoking conjugacy thereby reducing the number of interaction coefficients by half and gaining a doubling in computational speed. The $k=48$ case 
presented (see Figure 1) has 7,213 components and we also have 7 sets of $15,260,713$ non-zero interaction coefficients to be summed over. As the QDIA is non-Markovian long time integrations represent a significant computational challenge.

The restart procedure $[5,6]$ allows the potentially long time history integrals to be periodically truncated and the information about non-Gaussian correlations arising from the three-point term, and that of the off-diagonal elements (two-point term) to be estimated and added on in the new initial conditions. This procedure is employed at time intervals of sufficient length to capture the critical information about the off-diagonal elements while still reducing the computational challenge to a realistic task. For insufficiently long restart periods instabilities developed rapidly followed by CFL numerical instability. Importantly, in the regime of strong turbulence the presence of waves was not found to impact significantly on the performance of the cumulant update procedure. The spectral DNS code was found to have similar stability properties to the closure for a given time step.

\section{Results and discussion}

Figure 1 compares the zonally averaged kinetic energy $e\left(k_{x}\right)$ [2, Eqs. 5.1a, 5.2a] of the QDIA closure to DNS for flow over single realization mean topography with a particular random phase after a nondimensional evolution period of $t_{f}=0.2$. The DNS represents an average of 200 realizations whose phases are initially random whereas the QDIA employs a cumulant update method to render the time history integrals tractable at circularly truncated $k=48$ resolution in wavenumber space. In addition the QDIA closure makes use of a regularization procedure to properly represent all higher order terms. The details of the regularized CUQDIA closure are complex and are detailed in [7]. Put simply, regularization acts to restrict the range of the triad interaction terms via a cutoff parameter $\alpha$ where $\Theta\left(p-\frac{k}{\alpha}\right) \Theta\left(q-\frac{k}{\alpha}\right)$ acts on the $K(\mathbf{k}, \mathbf{p}, \mathbf{q})$ 


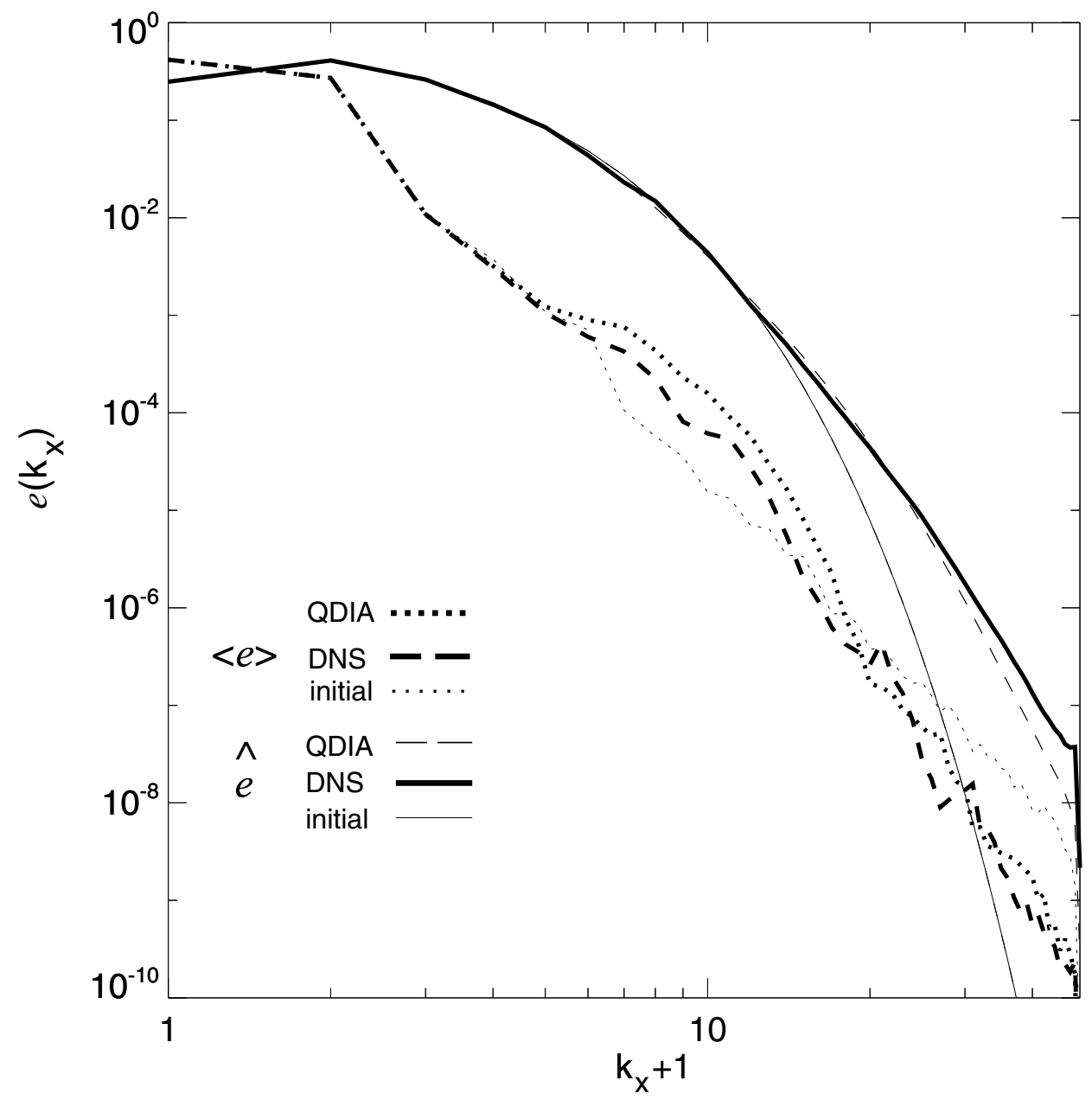

FiguRE 1: Zonally averaged mean $\langle e\rangle$ and transient $\hat{e}$ kinetic energy at C48 resolution for initial and final times. The zonal average sums over $k_{y}$ for a given $k_{x}$ in $e\left(k_{x}, t\right)$. 
and $A(\mathbf{k}, \mathbf{p}, \mathbf{q})$ interaction coefficients thereby ensuring consistency with Kolmogorov's hypothesis of local interactions in wavenumber space. Here, $\Theta$ is the Heavy side step function which vanishes for negative argument and is otherwise unity. Stability properties required that the minimum cumulant update period was $t=0.04$ corresponding to a restart at every 20 time steps. A regularization parameter of $\alpha=4$ was found to universally give the correct small scale behavior.

The nondimensional parameters we used are: time step $\Delta t=0.002$, viscosity $\hat{\nu}=0.0025, \beta=k_{0}^{2}=0.5$, large scale flow $U=0.06456$ corresponding to $15 \mathrm{~ms}^{-1}$, initial transient enstrophy spectrum $C_{\mathbf{k}}(0,0)=1.8 \times$ $10^{-1} k^{2} \exp \left(-\frac{2}{3} k\right)$, initial mean vorticity amplitude $\left\langle\zeta_{\mathbf{k}}(0)\right\rangle=k /\left(1+k^{3}\right)$ and topographic amplitude $h_{\mathbf{k}}=0.1 \times \sqrt{C_{\mathbf{k}}(0,0)}$. The DNS vorticity, mean closure vorticity and topography have random phase. The initial fields have general similarities to those observed in atmospheric flows as does the spectrum of the topography which falls away rapidly at the small scales. Figure 2 shows the evolution of the skewness [7, Eq. 5.4d] which is a very sensitive measure of the small scale differences between closure and DNS where again we see close agreement. The final large scale Reynolds numbers are $R_{L}^{\text {QDIA }}\left(t_{f}\right)=282.85$ and $R_{L}^{\mathrm{DNS}}\left(t_{f}\right)=285.18$ respectively.

\section{Conclusion}

In conclusion, we have developed the QDIA closure theory for the interaction of mean fields with Rossby waves and two-dimensional turbulence on a generalized $\beta$-plane. The generalized $\beta$-plane model for the barotropic-vorticity equation allows us to establish a one-to-one correspondence between the dynamical equations and Rossby wave dispersion relations on the $\beta$-plane and on the sphere. Our examination of the performance of the regularized CUQDIA closure at $\mathrm{C} 48$ resolution, in comparison with the statistics of large ensembles of DNS at moderate resolution and moderate large scale Reynolds 


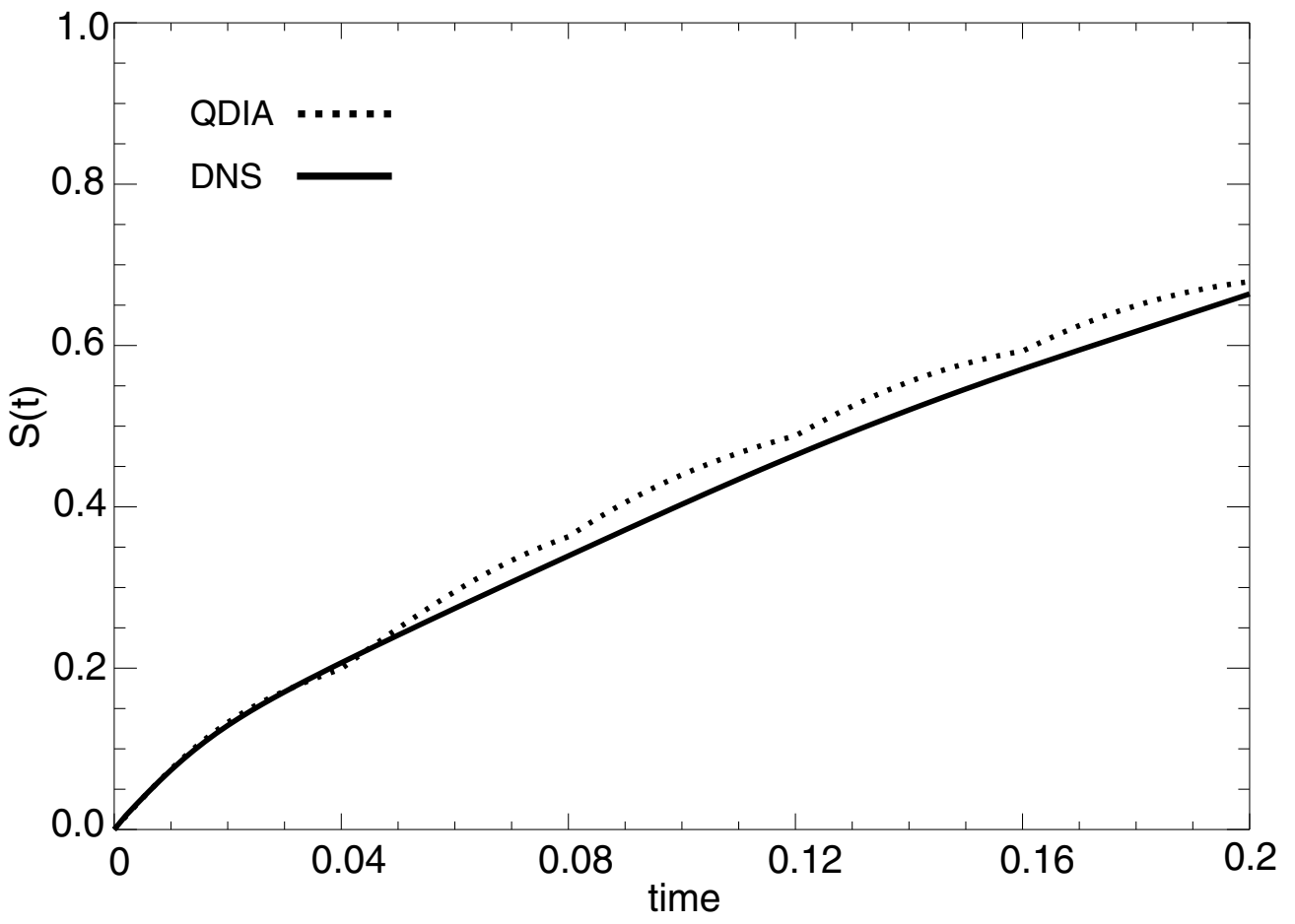

FiguRE 2: The skewness evolution.

number, found close agreement at all scales. The cumulant update or restart procedure performs well for restart evolution periods of $t=0.04$, although any significant reduction in restart time was found to generate instabilities and lead to CFL instability (not shown). In the regime of strong transients at the small scales and strong mean field at the largest scales, the cumulant update QDIA on the generalized $\beta$-plane was found to compare very closely to DNS with the restart procedure correctly handling large scale waves and strong small scale turbulence. 


\section{References}

[1] Frederiksen, J. S. 1999 Subgrid-scale parameterizations of eddy-topographic force, eddy viscosity, and stochastic backscatter for flow over topography. J. Atmos. Sci. 56, 1481-1494. C706, C710, C712, $\mathrm{C} 713$

[2] Frederiksen, J. S., \& O'Kane, T. J. 2005 Inhomogeneous closure and statistical mechanics for Rossby wave turbulence over topography $J$. Fluid Mech., In press C705, C706, C711, C713, C714

[3] Kraichnan, R. H. 1959a The structure of isotropic turbulence at very high Reynolds numbers. J. Fluid Mech. 5, 497-543. C705, C713

[4] Kraichnan, R. H. 1972 Test-field model for inhomogeneous turbulence. J. Fluid Mech. 56, 287-304. C711

[5] O'Kane, T. J., \& Frederiksen, J. S. 2003 Integro-differential closure equations for inhomogeneous turbulence. ANZIAM J. 44(E), C569-C589. http://anziamj . austms .org.au/V44/CTAC2001/Okan C707, C714

[6] O'Kane, T. J., \& Frederiksen, J. S. 2004 A tractable inhomogeneous closure theory for flow over mean topography. ANZIAM J. 45(E), C135-C148. http://anziamj . austms.org.au/V45/CTAC2003/Okan C707, C714

[7] O'Kane, T. J., \& Frederiksen, J. S. 2004 The QDIA and regularized QDIA closures for inhomogeneous turbulence over topography. J. Fluid Mech. 504, 133-165.

http://dx.doi.org/10.1017/S0022112004007980 C706, C710, C712, C713, C714, C716 